TITRE: VIVRE UNE EXPÉRIENCE ESTHÉTIQUE AU COLLÉGIAL : QUESTIONNEMENTS THÉORIQUES ET MÉTHODOLOGIQUES D'UNE RECHERCHE EXPLORATOIRE

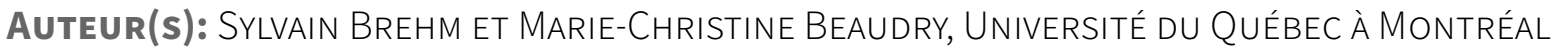

Publication: Discours, usAges, tRACES DE L'EXPÉRIENCE ESTHÉTIQUE EN CONTEXTE SCOLAIRE. PERSPECTIVES CROISÉES.

Directeurs: MARIE-Christine Beaudry, Sylvain Brehm et JeAn-FrançoIs Boutin

PAGES: $6-16$

URI: HTTP://HDL.HANDLE.NET/11143/14638

DOI: HTTPS://DOI.ORG/10.17118/11143/14638 


\section{Vivre une expérience esthétique au collégial : questionnements théoriques et méthodologiques d'une recherche exploratoire}

Sylvain Brehm, Professeur, Université du Québec à Montréal Marie-Christine Beaudry, Professeur, Université du Québec à Montréal

Résumé : Ce chapitre fait état d'une recherche exploratoire portant sur des dispositifs didactiques mis en place dans une classe de littérature auprès d'étudiants de niveau collégial. La finalité annoncée de ces dispositifs est à la fois de faire vivre aux étudiants des expériences esthétiques par la réception et la production d'œuvres littéraires, et de favoriser l'acquisition de savoirs et de compétences analytiques. Au travers des observations menées en classe et des entretiens réalisés auprès des étudiants, nous souhaitions notamment décrire ces dispositifs ainsi que les expériences vécues par les étudiants. La collecte de données a cependant généré plus de questions que de résultats. Nous présentons les principales hypothèses théoriques et méthodologiques que nous dégageons de nos analyses et réflexions.

Mots-clés : Expérience esthétique, réception et production d'œuvres littéraires, enseignement collégial, slam 


\section{L'enseignement de la littérature au collégial et l'expérience esthétique}

Depuis une quinzaine d'années, nombreux sont les chercheurs à dénoncer l'effet néfaste de l'excès de formalisme et de technicisme dans l'enseignement pré-universitaire de la littérature. Cette remise en question émane aussi bien des didacticiens (Chabanne, Dunas et Valdivia,, 2004; Dufays, 2007; Langlade et Fourtanier, 2007) que des spécialistes des études littéraires (Jouve, 2010; Schaeffer, 2011; Todorov, 2007). Leur constat est assez unanime : l'école peine encore à revaloriser les dimensions affective et émotionnelle de la lecture (Baroni et Rodriguez, 2014; Rouxel et Langlade, 2004; Vernay, 2013) et à faire vivre des expériences esthétiques (Rouxel, 2014) aux élèves.

Au Québec, cette difficulté se manifeste plus particulièrement au niveau collégial, où la conciliation entre le déploiement d'une posture réflexive et la manifestation d'une réaction subjective aux textes constitue souvent un défi pour les enseignants. Le problème tient en grande partie à la nature des exigences ministérielles qui mettent exclusivement l'accent sur l'acquisition de compétences analytiques et scripturales. Le développement de ces compétences, s'il permet une compréhension fine des œuvres, se réalise souvent en occultant les enjeux, les jugements, les émotions, les sensations, en somme les expériences - ou non expériences - esthétiques des lecteurs. C'est souvent l'une ou l'autre des postures de lecture qui est mise en place dans les dispositifs d'enseignement de la littérature comme si, d'emblée, les deux étaient concurrentes et ne pouvaient cohabiter. Dans ce contexte, l'expérience esthétique est rarement envisagée comme un objectif des activités d'enseignement. En outre, la rareté des expériences esthétiques vécues par les étudiants dans les classes de littérature du collégial n'est quasiment jamais problématisée et interrogée. Or, les savoirs et compétences analytiques ne devraient-ils pas être au service du partage et de l'explicitation de cette (non)expérience ? Ne serait-ce pas une opportunité pour les étudiants de donner un sens à leurs apprentissages?

Lorsqu'elle survient à l'école, l'expérience esthétique se produit dans un contexte spécifique, codifié, parfois contraignant et artificiel. Pour parvenir à saisir et définir ses caractéristiques, nous nous fondons à la fois sur les travaux des théoriciens littéraires (Genette, 1997), des philosophes européens et américains (Dewey, 2005; Kerlan, 2015; Rochlitz, 1994, 1995; Schaeffer, 2000, 2015), et des didacticiens en littérature et en arts (Chabanne et Villagordo, 2008). L'expérience esthétique est, selon eux, une expérience sensible (sensorielle et émotionnelle) et cognitive, à la fois singulière, subjective (Genette, 1997; Schaeffer, 2000, 2015) et collective (Rochlitz, 1994, 1995), issue de la production ou de la réception d'une œuvre. En nous inscrivant dans la conception de Dewey, nous faisons l'hypothèse que c'est entre autres par les expériences esthétiques multipliées que nous pouvons contribuer à donner du sens aux apprentissages faits en lecture et en écriture, l'objectif en contexte scolaire n'étant pas de donner une suprématie aux émotions, aux sens et au plaisir, mais plutôt de permettre aux élèves d'attribuer un sens à la relation qu'ils entretiennent avec les œuvres, que ces dernières soient lues ou encore produites par les élèves eux-mêmes. L'expérience de lecture-écriture littéraire, même menée en classe, peut ainsi ouvrir sur une expérience esthétique, celle-ci constituant une dimension de l'expérience de lecture-écriture littéraire.

L'observation et le partage d'une telle expérience passent par une médiation multimodale : « de soi à soi, de soi à l'autre [...] par du verbal, de l'écrit, par de l'iconique, du graphique (schéma, croquis...), par du corporel (mimer...) » (Chabanne et Villagordo, 2008, p. 10). Le rôle de l'enseignant est alors tout en finesse, à la fois guide, facilitateur et médiateur de la réception des élèves. Il les conduit tour à tour à observer attentivement, à décrire leur observation mais aussi « leur parcours » dans l'œuvre rencontrée (Lemonchois, 2012), à se questionner et questionner l'œuvre pour éventuellement construire du sens et interpréter l'œuvre. II les amène à réagir, puis à réfléchir aux effets que le texte - lu ou produit - suscite en eux. 


\section{La rencontre et la recherche menée}

Munis de ces quelques constats et réflexions théoriques, nous avons rencontré une enseignante de littérature du collégial. Elle poursuit, par son enseignement, deux objectifs principaux. D'une part, favoriser l'épanouissement et l'expression subjective et critique de ses étudiants en cherchant à leur faire vivre des expériences esthétiques; d'autre part, tenter de renouveler leur rapport à la littérature et le rendre signifiant pour eux. Pour ce faire, elle met en place cinq dispositifs d'enseignement-apprentissage articulés autour d'une ou plusieurs œuvres, dont la première finalité est de faire vivre des expériences esthétiques par la réception et la production d'œuvres littéraires, la seconde étant de permettre aux étudiants d'acquérir des savoirs et des compétences analytiques. Ajoutons que, selon l'enseignante, ses étudiants sont en difficulté en français écrit et que nombre d'entre eux semblent avoir un rapport parfois conflictuel avec la littérature telle qu'elle leur a été souvent présentée et enseignée dans leur cursus scolaire. Selon ce qu'elle a remarqué, les dispositifs mis en place semblent avoir des effets positifs sur eux.

Forts de cette rencontre, nous avons entrepris une recherche exploratoire. Nous souhaitions voir les effets des dispositifs didactiques mis en place par l'enseignante sur des étudiants du collégial. Plus spécifiquement, nous cherchions à 1) décrire ces dispositifs didactiques; 2) en décrire les effets sur les étudiants 3) cerner, dans les dispositifs, les éléments qui contribuent à susciter ces effets.

Nous avons suivi 22 étudiants de niveau collégial dans une classe de littérature dite « jumelée » d’un collège de la banlieue nord de Montréal. Ce type de classe a pour particularité d'accueillir des étudiants (17-18 ans) majoritairement francophones présentant des difficultés en français écrit. Ils bénéficient, à ce titre, de trois périodes de cours supplémentaires hebdomadaires ( 7 heures au lieu de 4 heures par semaine). Les étudiants sont au début de leur parcours au collégial.

Tous les enseignants des classes jumelées travaillent de concert à la mise en œuvre de cinq projets pédagogiques novateurs qui structurent le calendrier de la session (d'une durée de 15 semaines). Pour notre part, nous avons amorcé la recherche en suivant le déroulement d'un premier dispositif centré sur le slam qui s'est déployé sur cinq semaines, bâti autour d'un album de Grand Corps Malade. Les grandes étapes du dispositif sont les suivantes (nous ne sommes pas intervenus dans l'élaboration ni dans sa mise en œuvre): 
1. présentation magistrale par l'enseignante du genre et de quelques slameurs, dont Grand Corps Malade

2. lecture et analyse des textes de Grand Corps Malade (individuellement, en équipe et en grand groupe)

3. atelier d'écriture et de performance de slam avec un slameur professionnel dans le café du collège

4. rédaction individuelle d'un slam selon une grille de création établie par l'enseignante

5. présentation en classe du slam écrit individuellement

6. échanges écrits et oraux, à partir d'une grille de correction reprenant les éléments de la grille de création, avec les pairs et l'enseignante sur le slam écrit

7. réécriture individuelle du slam en fonction des réactions et commentaires des pairs et de l'enseignante

8. deuxième présentation en classe de la version retravaillée des slams et sélection de trois slams « coups de cour » par classe

9. présentation de tous les coups de cœur des classes jumelées lors d'une soirée slam, animée par une slameuse professionnelle, dans la salle de spectacle du collège. Les parents et tous les étudiants des classes jumelées étaient conviés à cette soirée, où étaient présentés à la fois les slams vainqueurs des classes, les slams d'un gagnant de l'année précédente et les slams de la slameuse professionnelle.

10. analyse finale et individuelle, en classe, d'un texte de Grand Corps Malade

Dans ce dispositif, le postulat est que la lecture des slams (des pairs, de Grand Corps Malade), la création personnelle d'un slam, l'écoute des slams lors de la soirée slam, la participation (en tant qu'acteur ou spectateur) au spectacle en marge de la classe peuvent générer une expérience esthétique. En parallèle, l'analyse finale de même que le slam écrit sont supposés refléter la compréhension et l'interprétation des textes - voire du genre - analysés.

Afin de répondre à nos objectifs, nous avons employé différents outils de collecte de données, soit l'observation, la tenue d'un journal par chacun des chercheurs et l'entretien semi-dirigé. En nous inscrivant dans une démarche de type ethnographique, nous avons observé in situ les étudiants et l'enseignante à plusieurs reprises pendant le déroulement du dispositif et en différentes circonstances : en classe, durant l'atelier avec le slameur, lors de la soirée slam. En parallèle à ces observations, des entrevues individuelles avec six étudiants ont été menées à différentes étapes du dispositif : au début, après la rencontre avec le slameur, après les échanges en équipe, après la deuxième prestation devant la classe, après la soirée slam. Enfin, un entretien en grand groupe, en classe, réalisé après la soirée slam a clos la collecte de données pour les étudiants.

Les questions des entretiens individuels portaient notamment sur les œuvres créées (quels choix, pourquoi, les étudiants se sentent-ils plus «créateurs»...), sur les slams lus et écoutés de leurs pairs (qu'ont-ils apprécié, pourquoi, influence sur eux), sur la rencontre avec le slameur lors de l'atelier (leurs impressions, ce qu'ils pensent en retirer, le rôle de l'artiste dans cette rencontre, le degré de difficulté associé à ce qu'ils ont fait, comment ils tiendront compte des commentaires et des propos du slameur), sur le rôle joué par les commentaires des pairs et de l'enseignant (les commentaires ont-ils aidé ou non, comment ont-ils tenu compte des commentaires des pairs). Les questions en grand groupe portaient sur la soirée slam (ce qu'ils ont apprécié, pourquoi ont-ils claqué des doigts, le lieu...). 
Enfin, tout au long du déroulement du dispositif, nous avons mené des entretiens semi-dirigés avec l'enseignante. Ils ont apporté des informations supplémentaires et complémentaires à nos observations afin de décrire le dispositif.

\section{Nos constats}

Notre enquête nous a rapidement confrontés à un problème fondamental : comment saisir un objet au caractère aussi polysémique que l'expérience esthétique? Il nous a ainsi fallu composer avec des obstacles d'ordre à la fois méthodologique et théorique (conceptuel).

L'une des premières difficultés à laquelle nous nous sommes heurtés a été, justement, la collecte de données. Certes, il s’agit d'un écueil fréquemment rencontré en recherche empirique. Il nous a été difficile de suivre durant cinq semaines les étudiants qui s'étaient montrés enthousiastes, au début de la recherche, pour participer à des entrevues tout au long de celle-ci. Des conflits d'horaires ou des absences de leur part ne nous ont pas permis de les rencontrer lors de toutes nos venues en classe. Par ailleurs, il est rapidement apparu difficile d'obtenir des réactions très élaborées, ce qui nous ramène à la difficulté de définir l'expérience esthétique : vers quoi diriger l'entretien? Comment susciter des réponses? Quels éléments de ces dernières identifier comme les indices, les traces du vécu d’une expérience esthétique?

Au terme des cinq semaines d'observations, de confrontations de journaux tenus par les deux chercheurs et d'échanges avec les étudiants, nous avons constaté que les données recueillies étaient peu nombreuses, qu'elles nous permettraient difficilement d'en dégager des résultats et qu'elles soulevaient finalement plus de questions qu'elles n’apportaient de réponses.

Ce constat nous a conduits à soulever plusieurs questions. Les difficultés rencontrées sont-elles imputables aux limites de nos observations et de notre analyse, qui ne nous auraient pas permis de déceler ou cerner des éléments pourtant pertinents dans les témoignages des élèves? Peut-on voir dans notre difficulté à observer des traces du vécu d'une expérience esthétique, dans le discours de ces derniers, la confirmation que «les cheminements par lesquels les lecteurs accèdent à l'expérience esthétique sont insaisissables » (Rouxel, 2014)?

À défaut de pouvoir apporter des réponses tranchées à ces questions, nous avons tenté de déterminer ce qui, dans notre protocole d'observation et d'analyse, dans la réponse des élèves aussi bien au dispositif mis en place par l'enseignante qu'à nos propres sollicitations, ainsi que dans le dispositif lui-même, pouvait expliquer les difficultés rencontrées lors de cette recherche.

\section{Nous}

Force est de reconnaître que notre enthousiasme, suscité par l'intention déclarée de l'enseignante (faire vivre une expérience esthétique aux élèves), nous a conduits à tenir pour acquis, d'une part, que notre conception de l'expérience esthétique était partagée par tous les acteurs (l'enseignante et ses élèves, l'enseignante et nous), d'autre part, qu'elle pouvait être mise en œuvre, qu'elle pouvait être « vécue » devant nous et, par le fait même, « observée ». Or, non seulement ce concept ne fait pas l'objet d'une définition unanime et consensuelle, mais, de plus, il apparaît rarement, sinon jamais, dans le discours des étudiants. Cela ne signifie toutefois pas nécessairement que ces derniers ne vivent jamais ce qui s'apparente à une expérience esthétique, mais tout simplement, par exemple, qu'ils nomment autrement ce type d'expérience, qu'ils ne lui accordent pas une valeur et une saillance particulières au regard de leur vécu quotidien, qu’ils ne savent comment exprimer la 
spécificité de leur expérience ou encore qu'ils ne ressentent pas le besoin de la partager (à tout le moins avec leur enseignante ou des chercheurs venus les interroger à ce propos).

Nous étions également convaincus que la nature du dispositif mis en œuvre par l'enseignante favoriserait des expériences esthétiques en classe, d'une part parce qu'il engage les étudiants dans des activités dont la finalité n’apparaît pas uniquement scolaire (rencontrer des artistes, lire et écrire dans des situations « authentiques » dans la mesure où la création de slams donne lieu à de véritables joutes), d'autre part parce qu'il convie ces mêmes étudiants à des activités hors de la classe. En d'autres mots, l'un des objectifs de l'enseignante est de décloisonner les pratiques de lecture et d'écriture privées et scolaires. Pourtant, rien ne garantit que l'intention de l'enseignante soit perçue et partagée par les étudiants. De plus, le dispositif, dans sa forme inhabituelle pour le collégial que dans son contenu, peut générer des expériences multiples, mais qui ne sont pas nécessairement esthétiques pour chacun des étudiants, peut-être encore moins dans l'immédiat. Nous avons cependant concentré notre regard de chercheurs sur la dimension esthétique, occultant possiblement d'autres expériences qui peuvent avoir été vécues par les étudiants, mais que nous n’avons pas pu cerner.

\section{Eux}

Comme nous l'avons mentionné plus haut, l'observation des étudiants et, d’une manière générale, les réponses obtenues de leur part ne nous ont pas permis de dégager des résultats élaborés. Cela tient en partie à des difficultés d'ordre logistique (absences de certains étudiants à des cours auxquels nous avons assisté, manque de temps pour les rencontrer en entrevue). De plus, l'observation des étudiants nous a permis de relever qu'ils interagissaient peu en classe, répondaient brièvement aux questions de l'enseignante ou aux interventions de leurs pairs. Ils nous sont apparus calmes en classe, réalisant les différentes activités proposées par l'enseignante ou le slameur selon les consignes données. En ce sens, ils semblent avoir joué le jeu de l'élève scolaire : présents en classe, effectuant les tâches demandées. Cependant, nous pouvons nous interroger sur leur réelle participation (Guetzkow, 2002). Ont-ils été déstabilisés par les différentes interventions de l'enseignante et du slameur, par les tâches à réaliser, par notre présence ? Au contraire, ont-ils plutôt retrouvé un cadre, et surtout des exigences, qui diffèrent finalement peu de ce à quoi ils ont été habitués dans leur cursus scolaire, c'est-à-dire écrire un texte et analyser une œuvre? Leur difficulté en français écrit, et plus largement leur rapport parfois conflictuel avec la littérature, voire avec l'école, peut-il avoir mis potentiellement « hors jeu » certaines réactions? Ce sont autant de questions soulevées pour lesquelles nous n’avons pas de réponse.

En outre, les étudiants que nous avons interrogés individuellement ont souvent exprimé des idées qui, de prime abord, semblent contradictoires: « oui j'ai aimé cela [les activités de création], mais je n’ai pas eu de plaisir »; « le projet m'a fait comprendre l'importance d'écrire, de toucher les gens par l'écriture [... ] non, le projet ne m’incite pas à lire ou à écrire plus, ou à écrire ou à lire, j'aime bien Patrick Sénécal comme lecture extrascolaire parce que les histoires sont plausibles (sic)». Réunis en grand groupe, les étudiants ont fourni des réponses encore moins nombreuses et moins développées : "c’était intéressant », "ça ne m’a pas dérangé de faire ça », «j'ai aimé les figures de style ». Pourtant, plusieurs d'entre eux ont exprimé des marques d'appréciation positive trois jours auparavant, lors de la soirée slam. Même lorsqu'invités à élaborer davantage par des relances, des reformulations, les étudiants interrogés ne se sont exprimés que fort peu. Est-ce la gêne de prendre la parole devant des chercheurs? Est-ce par crainte de dévoiler leurs réactions devant nous, parfois devant leurs pairs? Est-ce par difficulté à identifier et nommer ce qu'ils ont vécu? Il n'en demeure pas moins que les étudiants nous ont dit avoir été intéressés par la découverte de la création de leurs pairs, la nouveauté de l'activité, la rencontre avec l'artiste en dehors de la classe lors de l'atelier et la possibilité de se percevoir comme des individus et non seulement comme des élèves. La parole s'est libérée également un peu plus quand ils ont parlé des lieux : la 
rencontre avec le slameur dans un café du collège, la soirée avec tous les groupes dans une salle de spectacle sont des endroits qui les ont sortis de leur routine habituelle des classes, ce qu'ils ont apprécié. Cela nous amène à nous interroger sur l'importance du rôle du lieu dans l'expérience esthétique et, conséquemment, sur la possibilité de vivre une expérience esthétique authentique en classe, dans un contexte qui, par définition, est imposé et formaté... La difficulté de démêler ce qui relève de problèmes d'ordre méthodologique et d'ordre théorique se pose de nouveau.

Les entrevues avec les étudiants ont également mis au jour un autre paradoxe. Bien qu'ils soient unanimes à déclarer faire confiance à l'artiste chargé de l'atelier de création, à dire que celui-ci les a encouragés à écrire et les a outillés, ils n'ont pas vraiment tenu compte de ses conseils lors de la création de leur propre slam. Leur principale préoccupation a été de répondre aux attentes supposées de leur enseignante. En ce sens, ils ont adopté une même posture scolaire face à l'artiste et à l'enseignante. D'ailleurs, les réponses évoquées plus haut révèlent, justement, que nous n'avons probablement eu accès qu'aux « sujets scolaires ».

\section{Le dispositif}

Certains constats peuvent-ils être imputables au dispositif lui-même ? Ce dernier permet-il vraiment le développement d'un sujet « esthétique »? La plupart des textes présentés en exemple, au début de la séquence, ont contribué à inscrire le slam dans un genre spécifique : le récit de soi. Cet effet d'exemplarité, peut-être involontaire, a effectivement suscité des textes égotistes, dans lesquels primaient les dimensions affective et émotionnelle. Par ailleurs, ce dispositif mobilise le français écrit comme médium. Or, comme nous l'avons signalé plus haut, les classes jumelées regroupent justement des étudiants faibles en français. En ce sens, les activités demandées ont-elles pu les confronter à leurs difficultés à manier la langue, en plus de leur donner le sentiment d'accomplir une autre tâche scolaire?

Plusieurs remarques sur le contrat didactique, dégagées de nos observations et des différents entretiens, s'imposent:

- Les tâches demandées aux étudiants présentaient un caractère contraignant, en particulier parce qu'elles ont été imposées aux étudiants. De même, l'appariement avec d'autres étudiants, lors du travail d'équipe, a été décidé par l'enseignante. En outre, s'exposer devant ses pairs et interpréter devant eux une création personnelle a pu générer de l'anxiété, sachant que ces étudiants sont à un âge où le jugement des pairs est important. Enfin, la grille de création n’appelait pas explicitement à la réflexivité, mais insistait plus sur la présence de certains procédés stylistiques dans le texte de création. À cet égard, le contrat didactique, s'il était clair pour l'enseignante, l'était-il pour les étudiants?

- L'enseignante a joué un rôle décisif, durant toute la séquence, en intervenant pendant le processus de création et en évaluant le texte final. D’ailleurs, tous les étudiants savaient que le processus et le produit de leur création étaient soumis à une évaluation formelle de l'enseignante. 
- La place et le rôle tenus par l'enseignante invitent à s'interroger sur ceux des étudiants, notamment sur leurs intentions d'écriture et sur le destinataire implicite de leurs textes. À cet égard, si nous avons pu observer quelques moments d'échanges sur les textes (des étudiants et de Grand Corps Malade), en revanche nous n'avons pas vraiment assisté à l'émergence d'une communauté de lecteurs, ni d'espaces de discussion sur les lectures subjectives des textes créés par les élèves et de ceux de Grand Corps Malade. Cela suscite des interrogations quant à la participation réelle des étudiants. Les invitait-on réellement à être partie prenante, à être plus que des sujets scolaires, dans la production et la réception? Même si la participation des étudiants était déterminante dans la création par exemple du slam, était-ce pour autant une participation authentique (Lemonchois, 2010) ? Jusqu’à quel point les étudiants sentaient-ils qu'ils pouvaient réellement prendre des décisions de créateur ou de lecteur? Ont-ils seulement perçu qu'ils y étaient invités?

\section{Conclusion}

Si nous avons relevé que certains étudiants « vivaient quelque chose », nous ne pouvons pour autant le qualifier. Est-ce possible, d'ailleurs? Doit-on le faire? Cette expérience, si elle revêt une dimension esthétique pour des étudiants, est-elle le fruit du hasard, survenant au détour d'une activité planifiée par l'enseignant (poursuivant un autre objectif) ou, au contraire, peut-elle nécessairement survenir d'une planification méthodique ? Peut-elle être une visée didactique ou doit-elle demeurer une retombée possible ? Tous les élèves n'ont pas les mêmes dispositions face aux deux versants de l'expérience esthétique, la production et la réception. Par ailleurs, ce n'est pas parce qu'ils n'expriment pas, peu ou mal leur réception en contexte scolaire qu'ils ne vivent pas des expériences esthétiques dans leur vie privée, ou même dans le contexte scolaire.

Comme le suggérait Troyas à propos de la formation artistique,

si tous les élèves n'auront pas à se livrer un jour aux plaisirs de la création personnelle, tous gagneront néanmoins à avoir été culturellement préparés aux plaisirs de la contemplation et de la participation aux décisions esthétiques. À ceux qui ont le goût de la pratique, donnons le goût du jugement. À ceux qui n'éprouvent que le besoin de contemplation, donnons-leur, certes, le goût de l'expression mais donnons leur aussi les moyens du jugement (1992, p. 4).

« [L]e talent se forme, l'inspiration s'acquiert, l'émotion se prépare » (Porcher, 1973, p. 9). Et si, plutôt que de chercher à décrire l'expérience (esthétique) telle que vécue par les étudiants, nous ne cherchions pas plutôt à (mieux) éduquer leur regard, à leur apprendre à imaginer, à leur apprendre à créer, bref, à leur donner une éducation esthétique? 


\section{Bibliographie}

Baroni, R. et Rodriguez, A. (2014). Les passions en littérature. De la théorie à l'enseignement. Études de Lettres, (295).

Chabanne, J.-C., Dunas, A. et Valdivia, J. (2004). Entre social, affects et langages, l'oeuvre comme médiation. Le français aujourd'hui, (145), 76-83.

Chabanne, J.-C. et Villagordo, É. (2008). Dire l'expérience esthétique : le développement conjoint des compétences culturelles, identitaires et langagières. Dans J. Aden (dir.), Apprentissage des langues et pratiques artistiques (p. 281-306). Paris : Le Manuscrit.

Dewey, J. (2005). L’art comme expérience. Paris : Gallimard.

Dufays, J.-L. (2007). Enseigner et apprendre la littérature aujourd'hui : pour quoi faire ? Louvain-la-Neuve, Belgique : Presses universitaires de Louvain.

Genette, G. (1997). L'œuvre de l'art, tome 2: La relation esthétique. Paris : Seuil.

Guetzkow, J. (2002). How the arts impact communities: An introduction to the literature on arts impact studies. Communication présentée à Taking the Measure of Culture Conference, Princeton University, New Jersey. Repéré à https://www.princeton.edu/ artspol/workpap/WP20\%20-\%20Guetzkow.pdf

Jouve, V. (2010). Pourquoi étudier la littérature? Paris : Armand Colin.

Kerlan, A. (2015). L'expérience esthétique, une nouvelle conquête démocratique. Revista Brasileira de Estudos da Presença, 5(2), 266-286.

Langlade, G. et Fourtanier, M.-J. (2007). La question du sujet lecteur en didactique de la lecture littéraire. Dans É. Falardeau, C. Fisher, C. Simard et N. Sorin (dir.), Didactique du français, les voies actuelles de la recherche (p.101-123). Québec : Les Presses de l’Université Laval.

Lemonchois, M. (2010). Un nouveau champ d'investigation : la participation des élèves à la création lors d'intervention d'artistes dans des écoles primaires. Communication présentée à Encounters on Education / Encuentros sobre Educación / Rencontres sur l'Éducation, (11), 99-114.

Lemonchois, M. (2012). Nouvelle définition de l'enfance et enjeux pédagogiques de l'appréciation de l'image dans la littérature jeunesse. Actes du colloque Autour de l'adulte de demain : développer l'enfant philosophe et critique par la littérature jeunesse. Repéré à http://www.banq.qc.ca/documents/activites/ colloque/2012-2013/enfant_litterature/Lemonchois.pdf

Porcher, L. (1973). L'éducation esthétique. Luxe ou nécessité. Paris : Armand Colin.

Rochlitz, R. (1994). Subversion et subvention. Art contemporain et argumentation esthétique. Paris : Gallimard.

Rochlitz, R. (1995). Le critiquable en esthétique. Dans Rencontres Place publique. L'esthétique des philosophes. Paris : Dis Voir.

Rouxel, A. (2014). De l'importance de l'expérience esthétique dans la formation du lecteur. Dans J. Van Beveren (dir.), Littérature, langue et didactique. Hommages à Jean-Louis Dumortier (p. 205-219). Namur, Belgique : Presses universitaires de Namur.

Rouxel, A. et Langlade, G. (2004). Le sujet lecteur. Lecture subjective et enseignement de la littérature. Rennes, France : Les Presses de l'Université de Rennes.

Schaeffer, J.-M. (2000). Adieu à l'esthétique. Paris: PUF. 
Schaeffer, J.-M. (2011). Petite écologie des études littéraires : pourquoi et comment étudier la littérature. Paris : Thierry Marchaisse.

Schaeffer, J.-M. (2015). L'expérience esthétique. Paris : Gallimard.

Todorov, T. (2007). La littérature en péril. Paris : Flammarion.

Troyas, A. (1992). Pour une véritable éducation esthétique. Tréma, 2. doi:10.4000/trema.2412

Vernay, J.-F. (2013). Pour un renouveau de l'émotion en littérature. Paris : Éditions Complicités. 